\title{
A New Approach to Ergonomic Physical Risk Evaluation in Multi-Purpose Workplaces
}

\author{
Andrzej Marek LASOTA
}

\begin{abstract}
The profile of human factors and ergonomics is rising. To provide an ergonomic work environment, it is necessary to pay special attention to the design and evaluation of the workplace. Traditional production work practices do not sufficiently take into account task variability in job design and assessment. Variations in task content, organizational work performance are seen due to the effective use of equipment, which enables higher levels of productivity to be reached. However, this variation has an impact on physical ergonomics risk factors and, consequently, on the risk of developing work-related musculoskeletal disorders. Variations can cause difficulties in assessing workers' exposure to ergonomic risk. This study aims to develop a framework for the evaluation of physical ergonomic risk in multi-purpose workplaces. The presented framework integrates the concepts of well-known observation techniques used in the assessment of physical ergonomic risk factors in the multi-purpose workplace. The research framework highlights a worker's exposure to the ergonomic risk and illustrates how these results can be used to find solutions in future ergonomic interventions. The framework's application is presented by illustrative case studies. Theoretical, managerial and practical features of the framework are discussed.
\end{abstract}

Keywords: ergonomics; framework; human factors; industry; physical risk factors; workplaces

\section{INTRODUCTION}

Human factors and ergonomics (HFEs) have been the core issue for industry for many years and their profile is rising. The International Ergonomics Association defined HFEs as "the scientific discipline concerned with the understanding of interactions among humans and other elements of a system, and the profession that applies theory, principles, data and methods to design in order to optimize human well-being and overall system performance [1]. Thus, in the meaning of this definition, specialists, engineers should incorporate ergonomics in the design and assessment process to adapt them to the needs, abilities and limitations of workers, taking into account both mental workload [2] and physical exertion. Many factors influence workload, including: workstation layout, job design, tasks and working methods, tools and their design [3], as well as the anthropometric characteristics of workers [4].

To ensure an ergonomic working environment, it is necessary to pay special attention to the design [5] and evaluation of the workplace [6]. The productivity of workers is affected by physical ergonomic risk factors. Ergonomic risk factors manifest themselves in the form of work-related musculoskeletal disorders (WRMSDs), which are a common cause of health problems and ultimately sick leave in many professions. And musculoskeletal disorders are a common and serious problem in many industry sectors [7-9]. The major physical ergonomic risk factors for WRMSDs are: awkward posture, repetition, excessive physical load, duration of movement and vibration $[10,11]$. And researchers report that disorders can result in decreased productivity, quality of work, and increased costs [12-14] and absenteeism [1517]. Hence the identification of ergonomic risk factors becomes a key element in industry. Musculoskeletal disorders today affect more than 100 million Europeans [18]. In the European Union, over the past 12 months, 20 million employees have complained of work-related health problems, and 80 million employees are exposed to factors that may have negative effects on physical health [19]. The cost of all absences represents around $2,1 \%$ to $3,1 \%$ of the European Union's gross domestic product [20], which is a financial burden. Furthermore, Gaskin and Richard [21] reported that the value of lost productivity due to pain in the United States ranged from \$ 299 to \$ 335 billion annually. The annual cost of WRMSDs to the Canadian economy is estimated to be around $\$ 20$ billion [22]. In the US, WRMSDs accounted for $32 \%$ of all absences from work in 2015 due to sickness [15]. In Great Britain, musculoskeletal disorders accounted for $39 \%$ of all work related illnesses in 2016 - 2017 and it is estimated that 8,9 million working days were lost due to disorders, which represents $35 \%$ of all days lost due to work-related ill health [16]. In Poland, the second most common reason for absence from work due to illness was diseases of the musculoskeletal system. And in 2016, 36 million working days were lost, which represents $15,3 \%$ of all absences [17].

To prevent WRMSDs, musculoskeletal workload should be at an acceptable level. This can be determined by appropriate assessment methods which can also determine the risk of the task causing WRMSDs [23]. A number of methods have been developed [24, 25]. They can be divided into observational techniques and direct measurement techniques. In direct measurement techniques, instruments are used to measure exposure to variables at work, while continuous monitoring of body posture is performed by a device connected to the employee [11]. These systems can provide large quantities of highly accurate data on a range of exposure variables. However, direct measuring systems require a considerable investment in the purchase of equipment and its maintenance and require employing highly skilled technical personnel to operate the equipment [26]. Observational techniques, on the other hand, are inexpensive and can be used in a wide range of workplaces [26], where using other methods of observing workers would prove difficult because of the caused disruption [27].

Researchers have developed a number of observational techniques to evaluate postural workload and risk of WRMSDs: Ovako Working Posture Analysing System (OWAS) [28], Rapid Upper Limb Assessment (RULA) [29], Rapid Entire Body Assessment (REBA) [30], Occupational Repetitive Action (OCRA) [31], Quick 
Exposure Check (QEC) [32] and Postural Ergonomic Risk Assessment (PERA) [33].

Researchers widely use risk assessment methods to evaluate jobs, health outcomes and risk to WRMSDs of occupational groups in the production and assembly systems $[8,9,11,27,34,35]$. However, many industrial jobs are multi-task in nature [36] and there are multipurpose workstations. But, in the industry, a wide range of technologies is applied in the production of the final product. The growing level of customization and shorter product life manifests itself in the form of smaller batch sizes. For the production of small batch sizes, flexible and efficient production systems are necessary [37]. This results in a variety of components being manufactured on the same machines and workstations after retooling / rearrangement, which in turn affects the variability of tasks and work content performed by operators. And next, it can have an impact and leads to variability in workload, position during work, repeatability, duration of movement, vibration and finally the level of exposure to musculoskeletal disorders [9]. This variability of tasks and work content in the multi-purpose workstations may cause problems with estimating exposure to physical ergonomic risk factors and identifying the sources of risk, which in turn makes it difficult or impossible to formulate the right recommendations and take appropriate corrective actions. Moreover, despite the many different approaches offered in literature for the ergo-level analysis of working tasks, managers need a new and easy-to-use postural analysis protocol that can support risk mitigation through analysis and accelerate the evaluation process [38]. Thus, the purpose of this research was to develop a framework for physical ergonomic risk evaluation in multi-purpose workplaces.

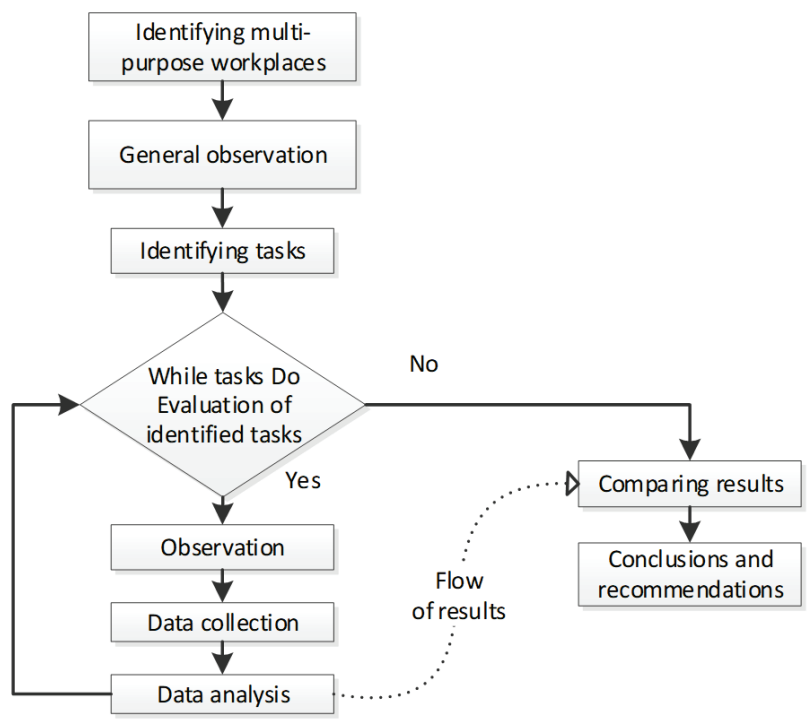

Figure 1 The flowchart of the framework

\section{RESEARCH METHOD}

The proposed new research approach is a framework Fig. 1. Research is preceded by an initial step related to the general observation and identification of multi-purpose workplaces. It gives insight and provides general information about multi-purpose workplaces and the tasks performed by employees. After this initial step, it is possible at the selected multi-purpose workplace to identify tasks and assess each task based on three major phases: 1) observation of a single task, 2) data collection, 3) data analysis.

In the next step, all findings collected from all evaluated tasks are correlated for comparison and analysis to determine the current state at the assessed multi-purpose workstation. This makes it possible to formulate conclusions and recommendations regarding the identified causes of abnormalities and risks, as well as opportunities for ergonomic improvement.

\subsection{Identifying Multi-Purpose Workplaces and General Observation}

The first crucial step is identifying workplaces where a variety of tasks is performed. After this initial selection process, chosen for evaluation are those multi-purpose workplaces where multiple tasks are performed at a single workstation and which meet the study criteria (e.g. the tasks are varied, affect the operator's posture, and involve repetitive movements). General visual observation and face-to-face interviews with managers, supervisors and technologists, as well as discussions, could be helpful in identifying and understanding multi-purpose workplaces. The gathered information should help the assessor understand the working condition and working environment, the job and the activities performed by the worker(s).

\subsection{Identifying Tasks}

Information collected as a result of observation of the worker(s) while performing tasks in the assessed workplace, group discussions and face-to-face interviews should be helpful and used to identify the tasks performed by the operator at the selected workstation. The information gathered in this step should be helpful in developing the evaluation plan, task evaluation order, data collection method(s), evaluation strategy, etc.

\subsection{While Tasks Do-Evaluation of Identified Tasks}

In this step, the while loop must be made for all identified tasks at the selected workstation and must include the following stages: observation, data collection and analysis.

\subsubsection{Observation}

Observation of workers during the performance of assessed task in the real workplace and face-to-face interviews with them should be used to explain any difficulties and problems related to the task being performed. Creating a friendly atmosphere and friendly rapport with employees should be helpful in conducting research.

\subsubsection{Data Collection}

It is possible to use several methods for data collection: observation, task analysis, and interview. Direct visual observation is a very useful and simple method, but video 
recording has many positive features. It does not interfere with the tasks performed by employees, allows for postevent analysis and can be repeated many times. Interviews and task analysis will depend on the research goal and assessment method(s).

\subsubsection{Data Analysis}

This stage is an in-depth analysis of all gathered data. The analysis of the risk level of musculoskeletal disorders and ergonomic risk factors should be carried out based on the evaluation plan, procedure for using WRMSDs method(s) and defined ergonomic criteria.

\subsection{Comparison of Results}

The study performed for each task in the workplace provides results related to exposure to ergonomic risk factors and WRMSDs of employees. They provide detailed information on workload, body positions, vibration and other risk factors. They report on harmless and harmful working postures and what risk factors (e.g. posture, force used) are at an unacceptable level. They also report the urgency of ergonomic intervention. The findings of the study and knowledge of action categories and action levels are major guidelines for the person conducting the study. The summary of all results of evaluated tasks gives additional information about action categories, action levels, ergonomic risk factors in relation to the operator's activities at the workplace. The comparison of the findings allows us to find out during which activities the worker is most exposed to physical risk factors and which of these activities are common to all evaluated tasks. This knowledge is necessary to propose the right solutions in order to take ergonomic corrective actions in the workstation.

\subsection{Conclusions and Recommendations}

At this final stage, after recognizing abnormalities (e.g. high level of vibration, force), it becomes possible to formulate conclusions regarding the exposure to risk to WRMSDs for each task. And it is possible to define the reasons of non-neutral body part positions and harmful action categories, action levels for which ergonomic intervention is required. These conclusions should be treated as guidelines and recommendations for improving working conditions and conducting ergonomic intervention. The knowledge obtained in the study, together with key ergonomics principles, should be used to develop a plan of corrective actions and ergonomic solutions that take into account the requirements arising from all tasks performed at the evaluated workstation.

\section{ILLUSTRATIVE CASE STUDIES}

3.1 Workplace for Manual Processing of Rubber Components-Case Study 1

The study was conducted in a company producing industrial fittings.

\subsubsection{Identifying Multi-Purpose Workplaces and General Observation}

General observation and face-to-face interviews, as well as discussions with the supervisor and observation in the field allowed the identification of multi-purpose workplaces. The selection of multi-purpose workplaces for evaluation was then conducted. More in-depth observation of the multi-purpose workstation in the factory, an additional face-to-face interview with the leader and information obtained from the operator and the leader led to a better understanding of the jobs and work activities performed by the employee.

A company performing manual processing of rubber components was selected for evaluation. The operator was 51 years old, with 3 years experience; in the workplace, the employee manually processed rubber elbows, compensators, and rubber feet, using a scalpel, scissors and a knife. The woman manually cut off the excess rubber that flowed from the mold under pressure from a hydraulic press.

\subsubsection{Identifying Tasks}

To identify tasks, observation of the worker in the workplace, discussion and previously obtained information was used. Three independent tasks performed in the workstation were identified: Task 1: rubber feet processing. The worker reached for a container from the cart, emptied the parts out onto the table top and set aside the empty container. Excess rubber from parts was then cut off, and the ready parts were put back into a container. After the operator was finished removing the excess rubber from the parts, the container was put back on a cart. Task 2: rubber compensators processing. The operator reached for two parts from the container located on the cart (one piece in one hand), put them on the table, cut off the excess rubber, and then put down the finished parts in the second cart. Task 3: rubber elbows processing. The employee reached for one piece with both hands from a pallet located on the floor, put it on the tabletop, cut off excess rubber, and then put the finished parts back down on the pallet.

The collected information was used to develop evaluation strategies and select an assessment method.

\subsubsection{Evaluation of Identified Tasks}

Observation, data collection and data analysis were performed for each of all of the identified tasks performed by operator at the selected workstation.

Observation

Observation of the employee took place during her daily routine work on current tasks in her real workplace and contained face-to-face interview. The operator was asked about tasks performed at the workstation. Thus, this additional information about the tasks was obtained and difficulties and problems with evaluated tasks, jobs and the workstation were explored.

Data collection

Data collection consisted of recording the worker's positions by visual observation. The pen-and-paper-based observational OWAS method with sampling interval (25 seconds) was used. The method is easy to use and allows 
for quick evaluation. The assessments were saved separately for each task. In total 212 work positions were assessed: 48 for task 1, 75 - task 2 and 89 - task 3 .

The OWAS method is based on a classification of different postures for the back (1 - neutral, 2 - bent forward, 3 - twisted, 4 - bent and twisted), arms ( 1 - both arms below shoulders, 2 - one arm above shoulders, 3 - both arms above shoulders), legs (1 - sitting, 2 - standing with both legs straight, 3 - standing with one leg straight, 4 - standing with one knee bent, 5 - standing with both knees bent, 6 kneeling, 7 - walking) and the force/load (1 - less than 10 $\mathrm{kg}, 2$ - between 10 and $20 \mathrm{~kg}, 3$ - more than $20 \mathrm{~kg}$ ) present during the task [28].

A four-digit code is applied to describe various combinations of body positions and force / load. These four-digit codes from different body parts in a specific position are combined with the estimated load to provide information about each postural load. For example, 4231 indicates that the worker's back is bent and twisted (back code: 4), working with one arm above the shoulder joint (arms code: 2), standing with one leg straight (legs code: 3 ), and handling a load weighing less than $10 \mathrm{~kg}$ (load code: 1). The combination creates categories describing the exposure to the risk of musculoskeletal disorders and action categories (AC) necessary to improve the working conditions: AC1 - no risk, intervention is not required; AC2 - low risk, immediate intervention is not required, but the ergonomic adjustment should be taken into account in future actions; AC3 - medium risk, ergonomic intervention should be carried out as soon as possible; AC4 - high risk, ergonomic intervention is required immediately.

\begin{tabular}{|c|c|c|c|c|c|}
\hline \multirow{2}{*}{$\begin{array}{c}\text { Posture } \\
\text { code }\end{array}$} & Task 1 & Task 2 & Task 3 & Total & \multirow[b]{2}{*}{$\mathrm{AC}$} \\
\hline & $\%$ & $\%$ & $\%$ & $\%$ & \\
\hline 1111 & 35,4 & 8,0 & - & 10,8 & 1 \\
\hline 1121 & - & - & 13,5 & 5,7 & 1 \\
\hline 1131 & - & - & 6,7 & 2,8 & 1 \\
\hline 1172 & - & - & 1,1 & 0,5 & 1 \\
\hline 1211 & - & 16,0 & - & 5,7 & 1 \\
\hline 1331 & - & - & 1,1 & 0,5 & 1 \\
\hline 2111 & 33,4 & 64,0 & - & 30,2 & 2 \\
\hline 2121 & - & - & 6,7 & 2,8 & 2 \\
\hline 2131 & - & - & 36,0 & 15,1 & 2 \\
\hline 2151 & 2,1 & - & - & 0,5 & 3 \\
\hline 2152 & - & - & 2,3 & 0,9 & 3 \\
\hline 2172 & - & - & 2,3 & 0,9 & 3 \\
\hline 2211 & 2,1 & 9,3 & - & 3,8 & 2 \\
\hline 2221 & - & - & 1,1 & 0,5 & 2 \\
\hline 2231 & - & - & 23,6 & 9,9 & 2 \\
\hline 2251 & - & 1,3 & - & 0,5 & 3 \\
\hline 3132 & - & - & 1,1 & 0,5 & 1 \\
\hline 3211 & 25,0 & - & - & 5,7 & 2 \\
\hline 3232 & - & - & 4,5 & 1,9 & 1 \\
\hline 4141 & - & 1,3 & - & 0,5 & 4 \\
\hline 4151 & 2,1 & - & - & 0,5 & 4 \\
\hline
\end{tabular}

\section{Data analysis}

The noted data were analysed separately for each task. And to each identified position a four-digit code was assigned and classified into harmless (AC1) and harmful (AC2, AC3, AC4) action categories.

For task 1, the author's results show that the most common four-digit position code was: 1111 - 35,4\% Tab. 1, classified into $\mathrm{AC} 1$; then $2111(33,4 \%, \mathrm{AC} 2)$ and 3211 $(25,0 \%, A C 2)$. Some $65 \%$ of positions were rated into harmful action categories. For task 2, the most common four-digit position code was $2111(64,0 \%$ and rated into $\mathrm{AC} 2)$, and 1211 (16,0\%, AC1). Some $76 \%$ of positions were rated into harmful action categories. In the case of task 3, $2131(36,0 \%, \mathrm{AC} 2), 2231(23,6 \%, \mathrm{AC} 2)$ and 1121 $(13,5 \%, \mathrm{AC} 1)$ were the most common four-digit postures. In this instance, $72 \%$ of the positions were rated into harmful action categories.

\subsubsection{Comparison of Results}

The most common four-digit posture code for all performed tasks was $2111,30,2 \%$, classified into AC2 and $2131,15,1 \%$, AC2. Of the positions, $72 \%$ were rated into harmful action categories. In total, two positions were rated into AC4; one 4141 (reaching for parts) in task 2 and one 4151 (putting ready parts back) in task 1 . Six positions were classified into AC3: one position coded 2151, which occurred during task 1 (reaching for a container with parts from the cart); two positions 2152 in task 3 (reaching for a part); two positions coded 2172 occurred during task 3 (transferring a part from the pallet); and one posture coded 2251 in task 2 (putting the part).

The comparison shows that positions classified into AC2 and coded as 2111 and 2211 were identified during two different tasks.

\subsubsection{Conclusions and Recommendations}

The findings show the differences among action categories for evaluated tasks and indicate awkward working positions. The common features of abnormality relating to working postures are activities involving reaching for a part and placing it down. And a common solution to this problem is needed. The recommendation is to use a manual forklift with height-adjustable lifts for the pallets, height-adjustable lifts for the containers, and to redesign the workplace layout - especially the location and orientation of containers.

\subsection{Semi-Automatic Spot Welding Workplace-Case Study 2}

The case study was conducted at an automotive company producing car parts. All steps from the framework Fig. 1 were performed. After identifying the set of multi-purpose workplaces, one of them was selected for evaluation. A workstation for semi-automatic spot welding of metal parts was chosen, where, after changing the tool in the machine and machine parameters, a second task was performed. The operator was 29 years old, with 11 years' experience on the job. The employee operated the machine at the welding workstation, which connected the metal parts using a resistance welding process. The parts were taken from the metal containers, placed in the handle of the machine, spot welded by the machine and after welding placed in other metal containers. Tasks performed by the operator consisted of a few steps and included: reaching for a part from the container, placing it in the welding instrument, reaching for one or more additional parts, putting them in a certain position, starting the welding machine and then transferring the welded parts to the container.

During observations and a face-to-face interview, two tasks were identified at the selected workplace: task 1 - 
welding two parts, task 2 - welding three parts. The evaluation was preceded by the separation of activities using hierarchical task analysis [39]. The OWAS method was used to evaluate the exposure to WRMSD risks [28]. Task 1 consisted of six activities, task 2 of 10 activities Tab. 2.

Table 2 OWAS score distribution and action categories (AC)

\begin{tabular}{|l|c|c|c|c|c|}
\hline \multicolumn{7}{|c|}{ Task 1 - welding two parts } \\
\hline \multicolumn{1}{|c|}{ Activities } & Back & Arms & Legs & Load & AC \\
\hline Reaching for a part & 4 & 1 & 1 & 1 & 2 \\
\hline Placing in the instrument & 1 & 1 & 1 & 1 & 1 \\
\hline Reaching for a second part & 1 & 1 & 1 & 1 & 1 \\
\hline Placing in the instrument & 1 & 1 & 1 & 1 & 1 \\
\hline Starting machine & 1 & 1 & 1 & 1 & 1 \\
\hline Placing welded part back & 4 & 1 & 1 & 1 & 2 \\
\hline \multicolumn{7}{|c|}{ Task 2 - welding three parts } \\
\hline Reaching for a part & 4 & 1 & 1 & 1 & 2 \\
\hline Placing in the instrument & 1 & 1 & 1 & 1 & 1 \\
\hline Reaching for two parts & 1 & 1 & 1 & 1 & 1 \\
\hline Placing in the instrument & 1 & 1 & 1 & 1 & 1 \\
\hline Starting machine & 1 & 1 & 1 & 1 & 1 \\
\hline Rotating part & 1 & 1 & 1 & 1 & 1 \\
\hline Reaching for two parts & 1 & 1 & 1 & 1 & 1 \\
\hline Putting in the instrument & 1 & 1 & 1 & 1 & 1 \\
\hline Starting machine & 1 & 1 & 1 & 1 & 1 \\
\hline Placing welded part back & 4 & 1 & 1 & 1 & 2 \\
\hline
\end{tabular}

For task 1 six postures were identified. In four cases the four-digit position code was 1111 and rated into AC1. In two cases, the four-digit posture code was 4111 and classified into AC2. For task 2 ten postures were identified. In eight cases the four-digit posture code was 1111, classified into $\mathrm{AC} 1$, whilst in two cases the four-digit posture code was 4111 (back code: 4 - back is bent and twisted; arms code: 1 - both arms below the shoulder joint; legs code: 1 - sitting position; load code: 1 - handling a load weighing less than $10 \mathrm{~kg}$ ) - classified into AC2.

Comparing the results of the evaluation of both tasks, it was found that in both cases the same four-digit posture code and action categories were present. A common issue for both evaluated tasks at the selected workplace was related to the back postures (bent forward and twisted) when reaching for the main parts from the container and laying the finished welded parts down into the second container. These identified poor back postures can lead to WRMSDs. Therefore, it was recommended to use heightadjustable lifts and tilt for containers, and to redesign the location and orientation of containers.

\subsection{A Workplace at the Cardboard Box Making Machine- Case Study 3}

The case study was carried out in a factory producing cardboard boxes. All steps from the framework were performed. A multi-purpose workstation at the semiautomatic cardboard box making machine was selected. After changing the tool in the machine and setting machine parameters, two types of cardboard boxes of various sizes are made from a cardboard sheet. Two tasks performed at the workstation have been identified: task 1 - one box per sheet, task 2 - five boxes per sheet. The operator was 37 years old, with 6 years of experience. His role was to retrieve the cut products from the machine, perform a visual inspection and palletize them. For the evaluation, the Postural Ergonomic Risk Assessment (PERA) [33] method was used. The PERA is observation-based and is a cube model method. It considers three parameters: posture $(P)$, force $(F)$, and duration $(D)$, which are divided into three levels of risk: low risk, medium risk, and high risk. The body segments and postures taken into account include: the trunk, shoulder, head and neck postures and other factors such as: elbow flexion, knee angle. Their exposure is associated with low risk, medium risk and high risk.

According to PERA, both tasks were divided into subtasks. Task 1 consisted of three subtasks, whilst task 2 included four subtasks. Task 1 included the following subtasks: picking up, visual inspection, palletizing. Task 2 included: picking up, dividing, palletizing, waiting. For task 1 the work subtask 1 score was 9 , the subtask 2 score was 6 and the subtask 3 score was 18 . The overall work cycle score A was 11 and indicated high risk, requiring an ergonomics intervention. In the case of task 2 the subtask 1 score was 4 , the subtask 2 score was 8 and the subtask 3 score was 9 , and the subtask 4 score was 1 . The overall work cycle score A was 5,5 and indicated possible risk Tab. 3.

Table 3 PERA indicator scores, subtask score and overall task score $A$ and risk

\begin{tabular}{|c|c|c|c|c|c|}
\hline \multicolumn{6}{|c|}{ Task 1 - one box per sheet } \\
\hline Subtasks & Posture & Duration & Force & $\begin{array}{c}\text { Subtask } \\
\text { score } \\
P \times D \times F\end{array}$ & $\begin{array}{c}\text { Overall work } \\
\text { cycle score, } \\
A\end{array}$ \\
\hline Picking up & 3 & 3 & 1 & 9 & \multirow{3}{*}{$\begin{array}{c}11 \\
\text { high risk }\end{array}$} \\
\hline $\begin{array}{l}\text { Visual } \\
\text { inspection }\end{array}$ & 3 & 1 & 2 & 6 & \\
\hline Palletizing & 3 & 3 & 2 & 18 & \\
\hline \multicolumn{6}{|c|}{ Task 2 - five boxes per sheet } \\
\hline Picking up & 2 & 1 & 2 & 4 & \multirow{4}{*}{$\begin{array}{c}5,5 \\
\text { possible risk }\end{array}$} \\
\hline Dividing & 2 & 2 & 2 & 8 & \\
\hline Palletizing & 3 & 3 & 1 & 9 & \\
\hline Waiting & 1 & 1 & 1 & 1 & \\
\hline
\end{tabular}

Comparing the results of the evaluation of both tasks, it was found that in both cases there were high risks associated with laying the cardboard boxes on the pallet. It was recommended to conduct training concerning correct working practices (working postures), use a manual forklift with height-adjustable lifts for pallets and redesign the orientation of the pallet.

\subsection{A workplace for Welding Structures-Case Study 4}

The case study was carried out in a factory producing metal structures. All steps from the framework were performed. A multi-purpose workplace for welding metal parts was selected. Two tasks performed at the workplace were identified: task 1 - welding, task 2 - grinding. The operator was 51 years old, with 33 years of experience. His job included welding parts of the metal structure - task 1; using an angle grinder to grind welds - task 2. For the evaluation the Quick Exposure Check (QEC) [32] method was used. The QEC is an observation technique that evaluates areas of ergonomic risk including: physical, organizational and psychosocial factors. It takes into account the repetitive movement and postures of four body parts: the back, shoulder / arm, wrist / hand and neck as well as additional factors (e.g. stress, work pace). In addition, the QEC takes into account the subjective exposure of employees. The result of QEC evaluation is exposure scores which are classified into four risk levels: 
low, moderate, high and very high for four body segments and additional factors. The total exposure level $E$ is calculated as a percentage rate between the actual total exposure score for body parts and the maximum possible total value. Initial action levels for the method are categorized into the following levels of exposure: low, medium, high, very high.

In reference to the QEC, the results of task 1's exposure level for the back, wrist / hand, and neck was high, whereas for the shoulder / arm it was medium. The overall exposure level $E$ indicated a high risk of WRMSDs requiring an ergonomics intervention. In the case of task 2 , a medium exposure level was observed for the back and shoulder / arm and a high exposure level for the wrist / hand and neck. The overall exposure level $E$ indicated a high risk of WRMSDs requiring an ergonomics intervention as soon as possible Tab. 4.

Table 4 QEC exposure level for body segments and overall exposure level $E$

\begin{tabular}{|c|c|c|c|c|c|}
\hline Tasks & Back & $\begin{array}{c}\text { Shoulder/a } \\
\text { rm }\end{array}$ & $\begin{array}{c}\text { Wrist/ } \\
\text { hand }\end{array}$ & Neck & $\begin{array}{c}\text { Overall } \\
\text { exposure } \\
\text { level, } E\end{array}$ \\
\hline Welding & high & medium & high & high & high risk \\
\hline Grinding & medium & medium & high & high & high risk \\
\hline
\end{tabular}

Comparing the results of both evaluated tasks, it was found that in both cases the overall exposure level $E$ was classified as high risk. The wrist / hand and neck body segments experienced a high exposure level. The main reason behind this was the incorrect height of the working area, which forced the operator to take awkward postures. It was recommended to conduct training concerning correct working practices (working postures), use a workbench with height-adjustable lifts in order to properly adjust the work space for the processed structure.

\section{CONCLUSIONS}

This study is aimed to develop a framework for physical ergonomic risk evaluation in multi-purpose workplaces. By integrating complementary approaches, the proposed method provides a more robust and holistic analysis, indicates risk factors, thus it prioritizes the improvements. The framework allows the identification of physical risk factors present both during each task and for duration of the entire job performed at the assessed workplace, supporting improvement actions.

The contribution of this study is multi-faced. Firstly, in theoretical terms, this approach provides a method that combines the assessment of physical risk of WRMSDs and supports the identification of risk factors in multi-purpose workplaces. The presented novel approach enables the measurement of a multi-purpose workplace's impact on postural workload and other physical ergonomics risk factors, and provides a better understanding of the physical ergonomic factors present in multi-purpose workplaces.

Secondly, in managerial terms, the study's outcomes establish a physical risk map for the company's multipurpose workstations, indicating critical points for improvement in both the short and long-term perspectives. This map also supports management in directing the employees' distribution according to job rotation, in order to minimize exposure to the physical risks of WRMSDs.
Thirdly, in practical terms, practitioners need to establish priorities for workplace interventions based on the assessments they have carried out. This framework is based on traditional pen-and-paper assessment methods, meaning that these techniques are more suited to the needs of practitioners. Practitioners usually have limited time and resources at their disposal for making assessments and are often faced with the challenge of preventing or reducing the risk of WRMSDs in the workplace. There is also a need for a basis for establishing priorities for intervention [26]. Exposure to risk factors of WRMSDs is an essential stage in the management and prevention program of WRMSDs, therefore practitioners need techniques to assess exposures that are easy and quick to use, sufficiently flexible to be applied to a range of jobs, and that are comprehensive and reliable for a range of risk factors. The proposed framework is easy to understand and apply and it is able to fully meet practitioners' expectations. Moreover, it can be used in the design phase to guarantee a certain ergo-quality level by using digital human modelling and methods such as OWAS or RULA in the virtual environment.

Finally, the framework presented in this study highlights the variability issues of tasks, their relationship with physical ergonomic risk factors and ultimately developing a link between physical risk factors and workplace safety by exploring the effects of tasks on the level of risk of WRMSDs. Moreover, it contributes at the individual as well as the organizational level, where its benefits can be seen in terms of employee health and wellbeing and workplace safety.

Despite the contribution of this study, further research is necessary to improve the efficiency of using the framework. In this study, only four multi-purpose workplaces were selected for evaluation from different factories. This was a consequence of the case studies being used to illustrate the proposed method. Therefore, further assessment is necessary to confirm the validity and reliability of the proposed framework. Moreover, further work and analysis is needed in order to test the framework on larger samples, including multi-purpose workplaces in a variety of industry sectors. A future study on the development of the framework should be directed at extending the framework to assess multi-purpose workplaces after ergonomic interventions in the context of continuous improvement.

\section{Acknowledgements}

The author would like to thank the managers, supervisors and employees for their cooperation and assistance in conducting the research.

\section{REFERENCES}

[1] IEA (2018). Definition and domains of ergonomics. http://www.iea.cc/whats/

[2] Eraslan, E., Can, G. F., \& Atalay, K. D. (2016). Mental workload assessment using a fuzzy multi-criteria method. TehnickiVjesnik-Technical Gazette, 23(3), 667-675. https://doi.org/10.17559/TV-20140401112509

[3] Vieira, E. R. \& Kumar, S. (2004). Working postures: a literature review. Journal of occupational rehabilitation, 14(2), 143-159. https://doi.org/10.1023/B:JOOR.0000018330.46029.05 
[4] Sumpor, D., Taboršak, D., \& Jurum-Kipke, J. (2015). Anthropometric Measures Important for Control Panel Design in Locomotive or Railcar. Tehničkivjesnik-Technical Gazette, 22(1), 1-10. https://doi.org/10.17559/TV-20140206135045

[5] Butlewski, M. (2014). Practical approaches in the design of everyday objects for the elderly. In Applied Mechanics and Materials, 657, 1061-1065. https://doi.org/10.4028/www.scientific.net/AMM.657.1061

[6] Lasota, A. M. \& Hankiewicz, K. (2017). The conceptual framework for physical risk assessment in multi-purpose workplaces. MATEC Web of Conferences, 137, p. 03007 , EDP Sciences. https://doi.org/10.1051/matecconf/201713703007

[7] Sanjog, J., Patel, T., Chowdhury, A., \& Karmakar, S. (2015). Musculoskeletal ailments in Indian injection-molded plastic furniture manufacturing shop-floor: mediating role of work shift duration. International Journal of Industrial Ergonomics, 48, 89-98. https://doi.org/10.1016/j.ergon.2015.04.004

[8] Lu, J. M., Twu, L. J., \& Wang, M. J. J. (2016). Risk assessments of work-related musculoskeletal disorders among the TFT-LCD manufacturing operators. International Journal of Industrial Ergonomics, 52, 40-51. https://doi.org/10.1016/j.ergon.2015.08.004

[9] Lasota, A. M. \& Hankiewicz, K. (2016). Assessment of risk to work-related musculoskeletal disorders of upper limbs at welding stations. International Symposium on Occupational Safety and Hygiene - SHO 2016, proceedings book, 138-140

[10] Musculoskeletal Disorders and Workplace Factors (1997).A critical review of epidemiologic evidence for work-related musculoskeletal disorders of the neck, upper extremity, and low back.

https://www.cdc.gov/niosh/docs/97-141/default.html

[11] Lasota, A. M. \& Hankiewicz, K. (2016). Working postures of spot welding machine operators. $12^{\text {th }}$ International Symposium on Occupational Safety and Hygiene of Portuguese Society of Occupational Safety and Hygiene (SHO), pp. 261-264. https://doi.org/10.1201/b21172-51

[12] Dunning, K. K., Davis, K. G., Cook, C., Kotowski, S. E., Hamrick, C., Jewell, G., \& Lockey, J. (2010). Costs by industry and diagnosis among musculoskeletal claims in a state workers compensation system: 1999-2004. American journal of industrial medicine, 53(3), 276-284. https://doi.org/10.1002/ajim.20774

[13] Bhattacharya, A. (2014). Costs of occupational musculoskeletal disorders (MSDs) in the United States. International Journal of Industrial Ergonomics, 44(3), 448454. https://doi.org/10.1016/j.ergon.2014.01.008

[14] Bevan, S. (2015). Economic impact of musculoskeletal disorders (MSDs) on work in Europe. Best Practice \& Research Clinical Rheumatology, 29(3), 356-373. https://doi.org/10.1016/j.berh.2015.08.002

[15] BLS (2016). Nonfatal Occupational Injuries and Illnesses Requiring Days Away from Work, 2015. https://www.bls.gov/news.release/osh2.toc.htm

[16] HSE (2018). Work Related Musculoskeletal Disorder Statistics (WRMSDs) in Great Britain 2017. http://www.hse.gov.uk/Statistics/causdis/musculoskeletal/m sd.pdf?pdf $=$ msd.

[17] ZUS (2018). Absencja chorobowa w 2017 roku. http://www.zus.pl/baza-wiedzy/statystyka/opracowaniatematyczne/absencja-chorobowa

[18] Lidgren, L., Gomez-Barrena, E., Duda, G. N., Puhl, W., \& Carr, A. (2014). European musculoskeletal health and mobility in Horizon 2020. Setting priorities for musculoskeletal research and innovation. Bone and Joint Research, 3(3), 48-50.

https://doi.org/10.1302/2046-3758.33.2000296
[19] De Norre, B. (2009). 8.6\% of workers in the EU experienced work-related health problems. Eurostat, Statistics in focus, 63, 2009.

http://www.bollettinoadapt.it/old/files/document/3369EUR OSTAT_20_08_2.pdf

[20] Schneider, E., Irastorza, X. B., \& Copsey, S. (2010). OSH in figures: Work-related musculoskeletal disorders in the EUFacts and figures. Office for Official Publications of the European Communities. https://osha.europa.eu/en/toolsand-publications/publications/reports/TERO09009ENC

[21] Gaskin, D. J. \& Richard, P. (2012). The economic costs of pain in the United States. The Journal of Pain, 13(8), 715724. https://doi.org/10.1016/j.jpain.2012.03.009

[22] McGee, R., Bevan, S., \& Quadrello, T. (2011). Fit for work?. In Musculoskeletal disorders and the Canadian labour market (Report 2). Ottawa: The Work Foundation, Conference Board of Canada.

[23] Roman-Liu, D., Bugajska, J., \& Tokarski, T. (2014). Comparative study of upper limb load assessment and occurrence of musculoskeletal disorders at repetitive task workstations. Industrial health, 52(6), 461-470. https://doi.org/10.2486/indhealth.2013-0232

[24] Takala, E. P., Pehkonen, I., Forsman, M., Hansson, G. Å., Mathiassen, S. E., Neumann, W. P., \& Winkel, J. (2010). Systematic evaluation of observational methods assessing biomechanical exposures at work. Scandinavian journal of work, environment \& health, 3-24. https://doi.org/10.5271/sjweh.2876

[25] Li, G. \& Buckle, P. (1999). Current techniques for assessing physical exposure to work-related musculoskeletal risks, with emphasis on posture-based methods. Ergonomics, 42(5), 674-695. https://doi.org/10.1080/001401399185388

[26] David, G. C. (2005). Ergonomic methods for assessing exposure to risk factors for work-related musculoskeletal disorders. Occupational medicine, 55(3), 190-199. https://doi.org/10.1093/occmed/kqi082

[27] Genaidy, A. M., Al-Shedi, A. A., \& Karwowski, W. (1994). Postural stress analysis in industry. Applied ergonomics, 25(2), 77-87. https://doi.org/10.1016/0003-6870(94)90068-X

[28] Karhu, O., Kansi, P., \& Kuorinka, I. (1977). Correcting working postures in industry: a practical method for analysis. Applied ergonomics, 8(4), 199-201. https://doi.org/10.1016/0003-6870(77)90164-8

[29] McAtamney, L. \& Corlett, E. N. (1993). RULA: a survey method for the investigation of work-related upper limb disorders. Applied ergonomics,24(2), 91-99. https://doi.org/10.1016/0003-6870(93)90080-S

[30] Hignett, S. \& McAtamney, L. (2000). Rapid entire body assessment (REBA). Applied ergonomics, 31(2), 201-205. https://doi.org/10.1016/S0003-6870(99)00039-3

[31] Occhipinti, E. \& Colombini, D. (1996). Alterazionimuscoloscheletrichedegliartisuperiori da sovaraccarico: metodi e criteri per l'inquardramentodell'esposizionelavorativa. $\mathrm{Med}$. Lav, 87.

[32] David, G., Woods, V., Li, G., \& Buckle, P. (2008). The development of the Quick Exposure Check (QEC) for assessing exposure to risk factors for work-related musculoskeletal disorders. Applied ergonomics, 39(1), 57 69. https://doi.org/10.1016/j.apergo.2007.03.002

[33] Chander, D. S. \& Cavatorta, M. P. (2017). An observational method for Postural Ergonomic Risk Assessment (PERA). International Journal of Industrial Ergonomics, 57, 32-41. https://doi.org/10.1016/j.ergon.2016.11.007

[34] Lasota, A. M. \& Hankiewicz, K. (2016). Evaluation of ergonomic risk in the production line of frozen food products. International Conference on Economics and Management Innovations - CEMI, ACSR-Advances in Computer Science Research, 272-278. https://doi.org/10.2991/icemi-16.2016.7 
[35] Lasota, A. M. \& Hankiewicz, K. (2017). The study of postural workload in assembly of furniture upholstery. MATEC Web of Conferences, 137, 07002. https://doi.org/10.1051/matecconf/201713707002

[36] Drinkaus, P., Sesek, R., Bloswick, D. S., Mann, C., \& Bernard, T. (2005). Job level risk assessment using task level ACGIH hand activity level TLV scores: a pilot study. International Journal of Occupational Safety and Ergonomics, 11(3), 263-281. https://doi.org/10.1080/10803548.2005.11076648

[37] Hallbeck, M. S., Bosch, T., Van Rhijn, G. J., Krause, F., De Looze, M. P., \& Vink, P. (2010). A tool for early workstation design for small and medium enterprises evaluated in five cases. Human Factors and Ergonomics in Manufacturing \& Service Industries, 20(4), 300-315. https://doi.org/10.1002/hfm.20222

[38] Savino, M., Mazza, A., \& Battini, D. (2016). New easy to use postural assessment method through visual management. International Journal of Industrial Ergonomics, 53, 48-58. https://doi.org/10.1016/j.ergon.2015.09.014

[39] Annett, J. (2004). Hierarchical task analysis. Handbook of Human Factors and Ergonomics Methods. 33-1 - 33-7. https://doi.org/10.1201/9780203489925.ch33

\section{Contact information:}

Andrzej Marek LASOTA, PhD, Eng.

(Corresponding author)

University of Zielona Gora

prof. Z. Szafrana 4 Street,

65-516 Zielona Gora, Poland

E-mails:am.lasota@o2.pl; a.lasota@iibnp.uz.zgora.p 